\title{
Serum carbonic anhydrase I and II autoantibodies in patients with chronic lymphocytic leukaemia
}

\author{
AHMET MENTESE, NERGIZ ERKUT ${ }^{2}$, SELIM DEMIR 3 , SERAP OZER YAMAN \\ AYSEGUL SUMER 5 , MEHMET ERDEM ${ }^{4}$, AHMET ALVER ${ }^{4}$, MEHMET SONMEZ ${ }^{2}$
}

\author{
${ }^{1}$ Program of Medical Laboratory Techniques, Vocational School of Health Sciences, Karadeniz Technical University, Trabzon, Turkey \\ ${ }^{2}$ Department of Haematology, Faculty of Medicine, Karadeniz Technical University, Trabzon, Turkey \\ ${ }^{3}$ Department of Nutrition and Dietetics, Faculty of Health Sciences, Karadeniz Technical University, Trabzon, Turkey \\ ${ }^{4}$ Department of Medical Biochemistry, Faculty of Medicine, Karadeniz Technical University, Trabzon, Turkey \\ ${ }_{5}^{5}$ Department of Nursing, School of Health Services, Recep Tayyip Erdoğan University, Rize, Turkey
}

\begin{abstract}
Cancer is the second most important cause of mortality, and millions of people either have or have had the disease. Leukaemia is one of the most common forms of cancer. Autoantibodies that have developed against the organism's self-antigens are detected in the sera of subjects with cancer. In recent years carbonic anhydrase (CA) autoantibodies have been determined in some autoimmune diseases and carcinomas, but the mechanisms underlying this immune response have not yet been fully explained. The purpose of this study was to determine CA I and II autoantibodies in subjects with chronic lymphocytic leukaemia (CLL) and to provide a novel perspective regarding the autoimmune basis of the disease. Autoantibody levels were investigated using enzyme-linked immunosorbent assay (ELISA) in serum samples from 37 patients with CLL and 37 healthy peers. Anti-CA I titres in the CLL group were significantly higher compared with the control group $(p=0.0001)$. However, there was no significant difference between CLL and control groups in terms of anti-CA II titres $(p=0.278)$. The prevalences of CA I and II autoantibodies in patients with CLL in this study were $27 \%$ and $24.3 \%$, respectively. Our results suggest that these autoantibodies may be involved in the pathogenesis of CLL. More extensive studies are now needed to reveal the entire mechanism.
\end{abstract}

Key words: autoantibody, cancer, carbonic anhydrase, chronic lymphocytic leukaemia.

(Centr Eur J Immunol 2018; 43 (3): 276-280)

\section{Introduction}

Chronic lymphocytic leukaemia (CLL) is characterised by the progressive accumulation of monoclonal lymphocytes with various phenotypes, such as $\mathrm{CD}^{+}, \mathrm{CD}^{+} 9^{+}$, $\mathrm{CD} 20^{\mathrm{dim}}, \mathrm{CD}^{2} 3^{+}$, and $\mathrm{SmIg}^{\mathrm{dim}}$ in peripheral blood, bone marrow, and lymphoid tissues [1]. CLL is the most common form of leukaemia in adults, representing 25-30\% of all leukaemias [2]. Approximately 18,960 new cases are diagnosed annually, and approximately 4660 deaths from CLL are predicted in adults in the USA in 2016. CLL is more common in men than in women, with a sex ratio of approximately $1: 3$ [3]. Compared with other chronic lymphoproliferative disorders, more severe immune disorders are seen in patients with CLL [2]. Autoimmunity is a well-known complication of lymphoproliferative diseases, in particular of CLL [4]. It has been suggested that leukaemia B cells are responsible for the production of autoantibodies in patients with CLL [5]. Induction of autoimmunity is characterised by the development of autoantibodies against a large number of autoantigens associated with malignant diseases. The presence of autoantibodies has been shown in the sera of patients with solid tumour and haematological malignancies [6]. The detection of these antibodies is expected to make an important contribution to the early diagnosis of some types of cancer [7-9].

Carbonic anhydrases (CAs) are vitally important enzymes responsible for the regulation of acid-base homeostasis in both healthy and pathological conditions. Members of the CA family contain 16 isoenzymes that differ from one another in terms of tissue distribution, cell localisation, catalytic activity, and resistance to inhibitors. These perform various functions, such as transport of carbon dioxide, $\mathrm{pH}$ regulation, ion transport, formation of stomach acidity, bone resorption, calcification, and tumorigenesis

Correspondence: Ahmet Mentese, PhD, Program of Medical Laboratory Techniques, Vocational School of Health Sciences,

Karadeniz Technical University, 61080, Trabzon, Turkey, e-mail: amentese028@ gmail.com

Submitted: 29.09.2016; Accepted: 18.12.2016 
during cancer cell development and invasion [10]. CA I and II are both cytosolic enzymes present in significant numbers in erythrocytes. CA I is the second most plentiful protein in erythrocytes after haemoglobin. CA II is a highly active isoenzyme involved in much total CA activity in a number of tissues. CA I and/or II autoantibodies have recently been demonstrated in various pathological conditions, such as autoimmune diseases (systemic lupus erythematosus, primary biliary cirrhosis, rheumatoid arthritis, and Sjögren's syndrome) and carcinomas (lung, colon, and prostate). However, the mechanisms underlying this immune response have not yet been fully explained [11-14]. The purpose of this study was to determine CA I and II autoantibodies in patients with CLL and to provide a novel perspective regarding the autoimmune basis of the disease.

\section{Material and methods}

\section{Study group}

Informed consent was obtained from all patients and controls, and approval for the study was granted by the Local Ethics Committee under reference no. 2016/30. Thirtyseven patients with untreated CLL in the A stage of the disease as classified by Binet, and thirty-seven healthy volunteers, were enrolled [15]. Clinical staging of the patient group was also defined according to Rai et al. [16]. The subtypes of CLL according to Rai scores (0-IV) were as follows: stage 0: $1(2.7 \%)$; stage I: 20 (54.1\%); and stage II: 16 (43.2\%). The study group consisted of 16 women and 21 men, with a mean age of $67.4 \pm 10.34$ years, and the control group comprised 15 women and 22 men with a mean age of $66.0 \pm 6.64$ years. Patients were selected from individuals presenting to the haematology clinic and referred from other practitioners. Patients with renal, coronary or liver failure, chronic inflammatory diseases, or anaemia, and subjects receiving chemotherapy or using oral contraceptives and anticoagulants were excluded from the study.

Five-millilitres blood samples from each individual were placed into vacutainer tubes without anticoagulant. These were then centrifuged at $1800 \mathrm{~g}$ for 10 minutes. Serum samples were stored at $-80^{\circ} \mathrm{C}$ until being used for measurements. Platelet count (PLT), lymphocyte count, haemoglobin $(\mathrm{Hb})$, haematocrit $(\mathrm{Hct})$, and lactate dehydrogenase (LDH) levels were determined using a Beckman Coulter autoanalyser. $\mathrm{CD}^{+}$and $\mathrm{CD} 19^{+}$analysis was performed using a flow cytometer (FACSCalibur, Becton Dickinson, East Rutherford, NJ).

\section{Determination of serum autoantibody to CA I and II}

Serum CA I and II autoantibodies were calculated using enzyme-linked immunosorbent assay (ELISA) method as previously described elsewhere [13, 17]. Briefly, flat- bottomed plates were coated with CA I or II $(10 \mu \mathrm{g} / \mathrm{ml})$ (Sigma-Aldrich, St.Louis, MO, USA) in carbonate buffer $(\mathrm{pH}=9.6)$. These were then incubated for 18 hours at $4^{\circ} \mathrm{C}$. In the next stage, the wells were washed four times with phosphate buffer (PBS) $(\mathrm{pH}=7)$ before being blocked with $3 \%$ skim milk in PBS at room temperature for $2 \mathrm{~h}$. The wells were then washed again four times with PBS containing $0.05 \%$ Tween-20 before incubation with $100 \mu \mathrm{l}$ of $1: 200$ diluted serum for $2 \mathrm{~h}$. Following these washing procedures, each individual well was incubated for $2 \mathrm{~h}$ with $100 \mu$ of a $1: 2000$ solution of peroxidase-conjugated anti-human IgG anti-serum (Sigma-Aldrich, St. Louis, MO, USA) in 3\% skimmed milk in PBS. A further five washes were performed with PBS containing $0.05 \%$ Tween-20, and the wells were then incubated with $100 \mu$ substrate solution for $20 \mathrm{~min}$. Reactions were halted by adding $100 \mu \mathrm{l}$ of $2 \mathrm{M}$ sulphuric acid to each well. The resulting absorbance was measured at $480 \mathrm{~nm}$ (Molecular Devices, CA, USA). Control wells containing no CA I or II were also employed for ELISA investigation of each serum studied. All assays were performed in duplicate. The specific binding of serum antibody to CA II was calculated as the mean absorbance of the antigen-coated wells minus the mean absorbance of the control wells. The results were expressed as absorbance units (ABSU).

\section{Statistical analysis}

Statistical analysis was performed on Statistical Package for the Social Sciences (Version 13.0, NY, USA) and MedCalc (Version 12.3, Mariakerke, Belgium) statistical software. Compatibility with normal distribution was determined using the Kolmogorov-Smirnov test. Data were shown as mean \pm standard deviation for normal distributed and median (interquartile range-IQR) for non-normal distributed variables. Differences between the two groups were analysed using Student's $t$-test for normally distributed data or Mann Whitney U test for non-normal distributed variables. Receiver operating characteristic (ROC) curves were used to determine the discriminatory dominance of CA I and II autoantibodies for the identification of CLL. Sensitivity, specificity, negative predictive values (NPV), and positive predictive values (PPV) were determined from ROC curves for autoantibodies of CA I, II, and other parameters. $p<0.05$ was regarded as significant.

\section{Results}

Thirty-seven CLL patients and 37 healthy subjects were included in this study. There was no significant difference in terms of mean age between the study and control groups $(p>0.05)$.

The mean absorbance value of anti-CA I antibodies for healthy subjects was $0.094 \pm 0.048$, and the absorbance was higher than 0.190 . The mean absorbance +2 SD of the 
Table 1. Clinical characteristics of the two groups

\begin{tabular}{|c|c|c|c|}
\hline & CLL $(n=37)$ & Control $(n=37)$ & $p$ \\
\hline Anti-CA I Ab (ABSU) & $0.154 \pm 0.086$ & $0.094 \pm 0.048$ & 0.0001 \\
\hline Anti-CA II Ab (ABSU) & $0.087 \pm 0.074$ & $0.072 \pm 0.030$ & 0.278 \\
\hline Haemoglobin (g/dl) & $13.1 \pm 2.08$ & $14.7 \pm 0.793$ & 0.0001 \\
\hline Haematocrit (\%) & $39.1 \pm 6.32$ & $44.6 \pm 1.83$ & 0.0001 \\
\hline Lymphocytes (cells/ul) & $\begin{array}{c}2290 \\
(1885-2770)\end{array}$ & $\begin{array}{c}37400 \\
(21550-50450)\end{array}$ & $0.0001^{*}$ \\
\hline Platelets (cells/ $\mu \mathrm{l})$ & $192865 \pm 84730$ & $229540 \pm 56490$ & 0.032 \\
\hline Lactate dehydrogenase (U/1) & $192 \pm 22.5$ & $434 \pm 181$ & 0.0001 \\
\hline $\mathrm{CD}^{+}(\%)$ & $85.6 \pm 21.2$ & & \\
\hline $\mathrm{CD}_{19}+(\%)$ & $86.8 \pm 12.0$ & & \\
\hline
\end{tabular}

CLL-chronic lymphocytic leukaemia

Data were expressed as mean $\pm S D$, median (interquartile range for $25-75 \%$ )

$p$ shows differences between control and CLL groups according to Student's t-test, "p shows differences between control and CLL groups according to MannWhitney $U$ test

Table 2. Receiver operating characteristic curve analysis of lymphocyte count, anti-CA I and II antibody levels, and their sensitivity, specificity, and positive and negative predictive values

\begin{tabular}{lcccccc}
\hline & AUC & $\begin{array}{c}\text { Cut-off } \\
\text { point }\end{array}$ & Sensitivity & Specificity & PPV & NPV \\
\hline Anti-CA I Ab & 0.721 & $>0.072$ & 95 & 43 & 61 & 80 \\
& $(0.605-0.819)$ & & $(79-98)$ & $(27-61)$ & $(47-74)$ & $(56-94)$ \\
\hline Anti-CA II Ab & 0.526 & $>0.047$ & 46 & 84 & 74 & 61 \\
& $(0.407-0.644)$ & & $(30-63)$ & $(68-94)$ & $(52-90)$ & $(48-75)$ \\
\hline Lymphocyte count & 0.993 & $>0.349$ & 100 & 97 & 97 & 100 \\
& $(0.938-1.00)$ & & $(91-100)$ & $(86-100)$ & $(86-100)$ & $(90-100)$ \\
\hline
\end{tabular}

Sensitivity, specificity, PPV and NPV values were expressed as \% within a 95\% CI

$A U C$ - area under the curve, $P P V$ - positive predictive value, $N P V$-negative predictive value

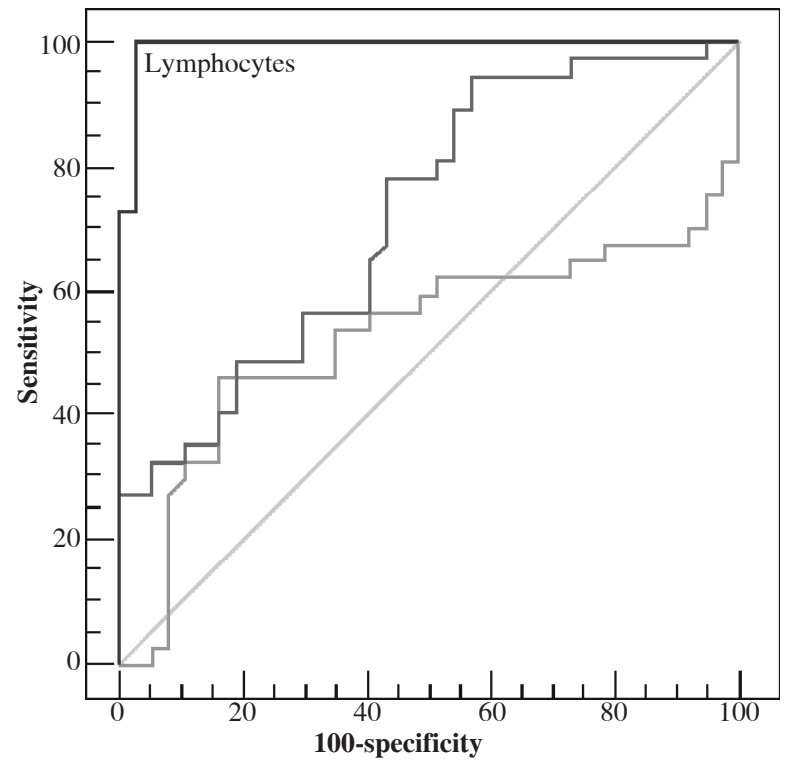

Fig. 1. Receiver operating characteristic curve analysis for all parameters in patients with chronic lymphocytic leukaemia healthy subjects was determined as positive. Positive results were obtained in 10 of the 37 cases with CLL. The mean absorbance value of the CLL group $(0.154 \pm 0.086)$ was significantly higher $(p=0.0001)$ than that of the healthy subjects (Table 1).

The mean absorbance value of anti-CA II antibodies for the healthy subjects was $0.072 \pm 0.030$, and the absorbance was higher than 0.132 . The mean absorbance $+2 \mathrm{SD}$ of the healthy subjects was also positive. Positive results were obtained in nine of the 37 cases with CLL. Values in one of the control subjects exceeded this cut-off level. The mean absorbance value of the CLL group $(0.087 \pm 0.074)$ was not significantly different to that of the healthy controls $(p=0.278)$ (Table 1$)$.

ROC curve analysis was also used to quantify lymphocytes, and anti-CA I and II antibody levels. Values for cutoff points, AUC, sensitivity, specificity, PPV, and NPV for individual parameters are shown in Table 2 and Figure 1.

\section{Discussion}

Cancer is the second most important cause of mortality, and millions of people either have or have had the 
disease. Leukaemia is one of the most common forms of cancer [3]. Various mutated or overexpressed proteins (such as tumour antigens) produce humoral and/or cellular responses in the immune system [18]. The potential relationship between autoimmunity and cancer is well known, and one of the forms of its expression is the development of autoantibodies. Determination of autoantibodies in cancer patients may be a first sign in the diagnosis of cancer [19]. Autoimmunity is a well-known complication of lymphoproliferative diseases, in particular of CLL [4]. There are several hypotheses concerning the source of autoantibodies. The simplest explanation is that the CLL clone is responsible for their production [20]. This study is the first report to show an increased autoimmune response to both CA I and II in the sera of CLL patients. Both CA I and II antibody titres were higher in the patients than in the controls ( $p=0.0001$ and 0.278 , respectively) (Table 1). In this study, an anti-CA I antibody cut-off level of 0.072 ABSU exhibited $95 \%$ sensitivity and $43 \%$ specificity, and an anti-CA II antibody cut-off level of 0.047 ABSU exhibited $46 \%$ sensitivity and $84 \%$ specificity based on ROC curve analysis (Table 2, Fig. 1). The prevalences of CA I and II autoantibodies in patients with CLL in this study were $27 \%$ and $24.3 \%$, respectively. The presence of autoantibodies against CA I and II has been observed in many pathological conditions, such as rheumatoid arthritis, recurrent pregnancy loss, acute anterior uveitis, Hashimoto's thyroiditis, Graves' disease, idiopathic chronic pancreatitis, and Sjögren's syndrome. The prevalence of CA I autoantibody is reported in the range of $5-38.1 \%$, and that of autoantibodies against CA II in the range of 5-61.9\% [13, 17, 21-23].

Protection of the acid-base balance is of considerable importance in tumorigenesis. Extracellular hydrogen ion concentrations in solid tumour are reported to be higher than those in normal neighbouring tissues $[11,24]$. Tumour cells express the ion transport protein, such as vacuolar-type $\mathrm{H}^{+}$-ATPase, $\mathrm{Cl}^{-} / \mathrm{HCO}_{3}^{-}$, and $\mathrm{Na}^{+} / \mathrm{H}^{+}$exchangers between the inner and outer regions of the cell, thus creating a $\mathrm{pH}$ gradient. Many tumour cells synthesise CAs that catalyse the production of $\mathrm{H}^{+}$and $\mathrm{HCO}_{3}^{-}$ions [11]. Sixteen different CA isoforms of the alpha family have been isolated and characterised in mammals [15]. CAs are currently the subject of significant research into carcinogenesis and tumour invasion. Studies have recently shown an incremental expression of specific cytosolic CA I and II in some carcinomas, including leukaemia [11, 24, 25]. CA I and II interact with various molecules due to their cellular locations, functions, and wide tissue distribution. These proteins are therefore a target molecule in the body. Autoantibodies against CAs have recently been demonstrated in many pathological conditions, such as cancer, and autoimmune and idiopathic diseases. Although the mechanisms involved have not been identified precisely, oxidative stress has been reported to be potentially signif- icant in the formation of these autoantibodies [12, 13, 21, $26,27]$. Oxidative stress results from acceleration of the rate of free radical formation and/or a decrease in the rate at which these are eliminated. In either condition a severe imbalance occurs between free radical formation and the antioxidant defence mechanism [28]. Increased reactive oxygen or nitrogen species (ROS/RNS) lead to tissue injury and compromise of numerous biomolecules, including proteins, nucleic acids, structural carbohydrates, and lipids. The reaction of ROS with lipids causes these molecules to undergo oxidative breakdown. Malondialdehyde (MDA) is an end-product of lipid peroxidation capable of being covalently bound to proteins, and especially to the $\varepsilon$-amino groups of lysine residues. These oxidative disturbances may influence the immune system, resulting in the development of specific autoimmune processes [29]. The lipid peroxidation end-product 4-hydroxy-2-nonenal (HNE) and MDA are known to alter proteins and to modify their antigenic properties [30]. One study of erythrocytes proved that CA II is the first target of HNE [31]. Numerous antiMDA-modified proteins have been detected in systemic diseases in previous studies, such as systemic lupus erythematosus, periarteritis nodosa, scleroderma, atherosclerosis, and rheumatoid arthritis. It has also been suggested that these autoantibodies may be of predictive value for systemic diseases [29, 32-35]. Studies have shown increased levels of oxidative stress parameters, such as MDA, superoxide anion, and nitric oxide, while decreased levels of the activities of antioxidant enzymes, such as superoxide dismutase (SOD), catalase (CAT), glutathione peroxidase (GPx), and glutathione reductase (GR) in the sera of patients with CLL $[36,37]$. In light of these data, we anticipated that oxidative by-products, including MDA, might generate the spread of neoantigens and confirm a potential association between autoimmunity and oxidative stress.

\section{Conclusions}

In conclusion, CA I autoantibody titres were significantly higher in subjects with CLL compared to the controls. More extensive studies are now needed to reveal the entire mechanism involved.

The authors declare no conflict of interest.

\section{References}

1. Hodgson K, Ferrer G, Montserrat E, et al. (2011): Chronic lymphocytic leukemia and autoimmunity: a systematic review. Haematologica 96: 752-761.

2. Ghia P, Ferreri AJM, Caligaris-Cappio F (2007): Chronic lymphocytic leukemia. Crit Rev Oncol Hematol 64: 234-246.

3. Siegel RL, Miller KD, Jemal A (2016): Cancer statistics, 2016. CA Cancer J Clin 66: 7-30. 
4. Mauro FR, Foa R, Cerretti R, et al. (2000): Autoimmune hemolytic anemia in chronic lymphocytic leukemia: Clinical, therapeutic, and prognostic features. Blood 95: 2786-2792.

5. Kipps TJ, Carson DA (1993): Autoantibodies in chronic lymphocytic leukemia and related systemic autoimmune diseases. Blood 81: 2475-2487.

6. Abu-Shakra M, Buskila D, Ehrenfeld M, et al. (2001): Cancer and autoimmunity: autoimmune and rheumatic features in patients with malignancies. Ann Rheum Dis 60: 433-441.

7. Chapman CJ, Murray A, McElveen JE, et al. (2008): Autoantibodies in lung cancer: possibilities for early detection and subsequent cure. Thorax 63: 228-233.

8. Zhong L, Ge K, Zu JC, et al. (2008): Autoantibodies as potential biomarkers for breast cancer. Breast Cancer Res 10: R40.

9. Lowe FJ, Shen W, Zu J, et al. (2014): A novel autoantibody test for the detection of pre-neoplastic lung lesions. Mol Cancer 13: 78 .

10. Gokcen T, Gulcin I, Ozturk T, et al. (2016): A class of sulfonamides as carbonic anhydrase I and II inhibitors. J Enzyme Inhib Med Chem 29: 1-9.

11. Leppilampi M, Koistinen P, Savolainen ER, et al. (2002): The expression of carbonic anhydrase II in hematological malignancies. Clin Cancer Res 8: 2240-2245.

12. Adamus G (2009): Autoantibody targets and their cancer relationship in the pathogenicity of paraneoplastic retinopathy. Autoimmun Rev 8: 410-414.

13. Alver A, Senturk A, Cakirbay H, et al. (2011): Carbonic anhydrase II autoantibody and oxidative stress in rheumatoid arthritis. Clin Biochem 44: 1385-1389.

14. Mentese A, Erkut N, Sumer A, et al. (2015): Anti-carbonic anhydrase antibodies in iron deficiency anemia. Hematology 20: 363-367.

15. Binet JL, Auquier A, Dighiero G, et al. (1981): A new prognostic classification of chronic lymphocytic leukemia derived from a multivariate survival analysis. Cancer 48: 198-206.

16. Rai KR, Sawitsky A, Cronkite EP, et al. (1975): Clinical staging of chronic lymphocytic leukemia. Blood 46: 219-234.

17. Kino-Ohsaki J, Nishimori I, Morita M, et al. (1996): Serum antibodies to carbonic anhydrase I and II in patients with idiopathic chronic pancreatitis and Sjögren's syndrome. Gastroenterology 110: 1579-1586.

18. Zhang JY, Casiano CA, Peng XX, et al. (2003): Enhancement of antibody detection in cancer using panel of recombinant tumor-associated antigens. Cancer Epidemiol Biomarkers Prev 12: 136-143.

19. Vedeler CA, Antoine JC, Giometto B, et al. (2006): Management of paraneoplastic neurological syndromes: report of an EFNS Task Force. Eur J Neurol 13: 682-690.

20. Duek A, Shvidel L, Braester A, et al. (2006): Clinical and immunologic aspects of B chronic lymphocytic leukemia associated with autoimmune disorders. Isr Med Assoc J 8: 828-831.

21. Alver A, Mentese A, Karahan SC, et al. (2007): Increased serum anti-carbonic anhydrase II antibodies in patients with Graves' disease. Exp Clin Endocrinol Diabetes 115: 287-291.

22. Karahan SC, Guven S, Mentese A, et al. (2009): Serum anti-carbonic anhydrase I and II antibodies and idiopathic recurrent pregnancy loss. Reprod Biomed Online 19: 859-863.

23. Turk A, Aykut M, Akyol N, et al. (2014): Serum anti-carbonic anhydrase antibodies and oxidant-antioxidant balance in patients with acute anterior uveitis. Ocul Immunol Inflamm 22: 127-132.
24. Demir C, Demir H, Esen R, et al. (2010): Erythrocyte catalase and carbonic anhydrase activities in acute leukemias. Asian Pac J Cancer Prev 11: 247-250.

25. Parkkila S, Lasota J, Fletcher JA, et al. (2010): Carbonic anhydrase II. A novel biomarker for gastrointestinal stromal tumors. Mod Pathol 23: 743-750.

26. Iuchi Y, Okada F, Onuma K, et al. (2007): Elevated oxidative stress in erythrocytes due to a SOD1 deficiency causes anaemia and triggers autoantibody production. Biochem J 402: 219-227.

27. Iuchi Y, Okada F, Takamiya R, et al. (2009): Rescue of anaemia and autoimmune responses in SOD1-deficient mice by transgenic expression of human SOD1 in erythrocytes. Biochem J 422: 313-320.

28. Dalle-Donne I, Scaloni A, Giustarini D, et al. (2005): Proteins as biomarkers of oxidative/nitrosative stress in diseases: the contribution of redox proteomics. Mass Spectrom Rev 24: 55-99.

29. Amara A, Constans J, Chaugier C, et al. (1995): Autoantibodies to malondialdehyde-modified epitope in connective tissue diseases and vasculitides. Clin Exp Immunol 101: 233-238.

30. Toyoda K, Nagae R, Akagawa M, et al. (2007): Protein-bound 4-hydroxy-2-nonenal: an endogenous triggering antigen of antI-DNA response. J Biol Chem 282: 25769-25778.

31. Uchida K, Hasui Y, Osawa T (1997): Covalent attachment of 4-hydroxy-2-nonenal to erythrocyte proteins. J Biochem 122: $1246-1251$.

32. Salonen JT, Ylä-Herttuala S, Yamamoto R, et al. (1992): Autoantibody against oxidised LDL and progression of carotid atherosclerosis. Lancet 339: 883-887.

33. Cvetkovic JT, Wallberg-Jonsson S, Ahmed E, et al. (2002): Increased levels of autoantibodies against copper-oxidized low density lipoprotein, malondialdehyde-modified low density lipoprotein and cardiolipin in patients with rheumatoid arthritis. Rheumatology (Oxford) 41: 988-995.

34. Ben Mansour R, Lassoued S, Elgaied A, et al. (2010): Enhanced reactivity to malondialdehyde-modified proteins by systemic lupus erythematosus autoantibodies. Scand J Rheumatol 39: 247-253.

35. Winyard PG, Ryan B, Eggleton P, et al. (2011): Measurement and meaning of markers of reactive species of oxygen, nitrogen and sulfur in healthy human subjects and patients with inflammatory joint disease. Biochem Soc Trans 39: 1226-1232.

36. Djurdjevic P, Zelen I, Ristic P, et al. (2006): Oxidative stress markers in chronic lymphocytic leukemia. Medicus 7: 52-56.

37. Ortin X, Giralt M, Romeu M, et al. (2012): Oxidative stress in patients with early stage chronic lymphocytic leukemia, assessment and correlation with prognostic factors. J Hematol 1: 77-88. 